\title{
Correction to: A New Rock Brittleness Index Based on the Peak Elastic Strain Energy Consumption Ratio
}

\author{
Fengqiang Gong ${ }^{1,2}\left(\mathbb{D} \cdot\right.$ Yunliang Wang ${ }^{3}$
}

Published online: 5 February 2022

(c) Springer-Verlag GmbH Austria, part of Springer Nature 2022

\section{Correction to: Rock Mechanics and Rock Engineering https://doi.org/10.1007/s00603-021-02738-y}

In the original publication, the author has missed to update a few errors and they are corrected as follows:

\section{Correction to text}

1. In the line 4 of "Abstract", "failure energy" should be "post-peak failure strain energy".

2. In the "List of Symbols", the descriptions of several symbols are incorrect, the correct ones are as follows:

$U_{\mathrm{p}}^{\mathrm{e}} \quad$ Peak elastic strain energy

$U^{\mathrm{f}} \quad$ Post-peak failure strain energy

$U_{\mathrm{r}}^{\mathrm{e}} \quad$ Residual elastic strain energy

$U^{\mathrm{a}} \quad$ Additional input strain energy

$U^{\text {ec }}$ Consumed elastic strain energy during the failure process

$U_{\mathrm{p}}^{\mathrm{d}} \quad$ Peak dissipated strain energy

3. In the line 8 of the first paragraph of Sect. 2, the "input energy" should be "input strain energy"; in the line 13, the "dissipated energy" should be "dissipated strain energy".

The original article can be found online at https://doi.org/10.1007/ s00603-021-02738-y.

Fengqiang Gong

fengqiangg@126.com

1 Engineering Research Center of Safety and Protection of Explosion and Impact of Ministry of Education (ERCSPEIME), Southeast University, Nanjing 211189, China

2 School of Civil Engineering, Southeast University, Nanjing 211189, China

3 School of Resources and Safety Engineering, Central South University, Changsha 410083, China
4. In the line 8 of the second paragraph of Sect. 2, the "failure energy" should be "post-peak failure strain energy"; in the line 10, the "failure energy" should be "post-peak failure strain energy"; in line 14, the "input energy" should be "input strain energy".

5. In the line 5 of the third paragraph of Sect. 2, the "peak elastic strain density" should be "peak elastic strain energy"; the "failure energy density" should be "postpeak failure strain energy"; in line 6, the "residual elastic strain energy density" should be "residual elastic strain energy"; in line 14, the "input energy" should be "input strain energy".

6. In the line 33 of the third paragraph of Sect. 3.1, the "pre-peak elastic strain energy" should be "peak elastic strain energy".

7. In the line 4 of the first paragraph of Sect. 3.2, the "energy density" should be "strain energy".

8. In the line 17 of Sect. 3.3, the "input energy" should be "input strain energy".

\section{Correction to Tables}

1. In the line 1 of column "Energy parameters" of Table 1, the "additional input energy" should be "additional input strain energy"; the "total failure energy" should be "post-peak failure strain energy".

2. In the line 5 of column "Energy parameters" of Table 1, the "peak dissipated energy" should be "peak dissipated strain energy".

3. In the lines 6 and 7 of column "Characteristics" of Table 1, the "additional input energy" should be "additional input strain energy".

4. In the lines 13,14 and 16 of column "Characteristics" of Table 1, the "dissipated energy" should be "dissipated strain energy".

5. In the lines 22 and 24 of column "Characteristics" of Table 1, the "fracture energy" should be "post-peak failure strain energy". 
6. In the line 27 of column "Characteristics" of Table 1, the "additional input energy" should be "additional input strain energy".

7. In the lines 34 and 35 of column "Characteristics" of Table 1, the "failure energy" should be "post-peak failure strain energy".
8. In the line 38 of column "Characteristics" of Table 1, the "input energy" should be "input strain energy".

\section{Correction to Figures}

1. Figure $1 \mathrm{~b}$ of the original article is incorrect. The corrected version is shown below.

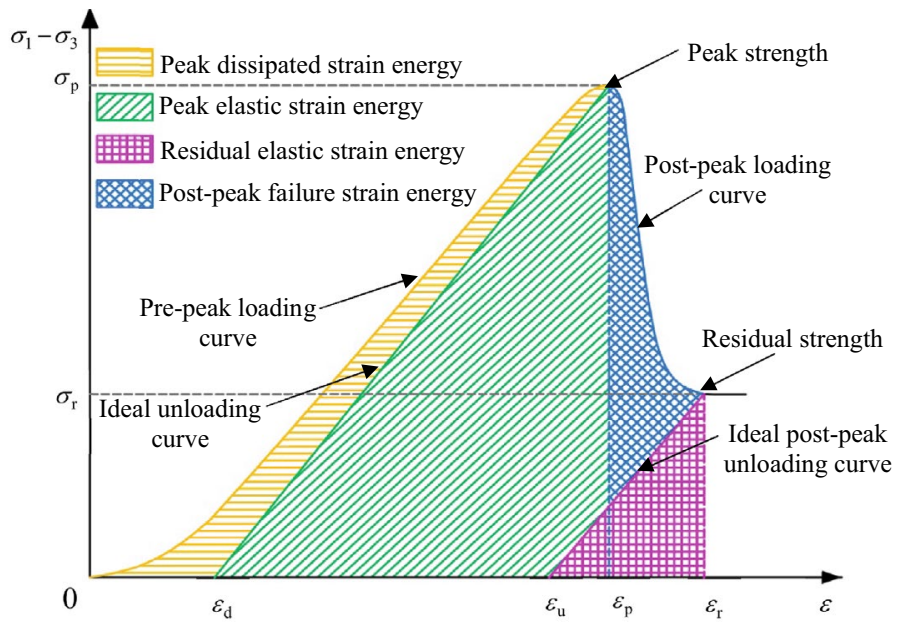

(b) Sketch of the energy evolution characteristics during the rock deformation and failure process (After Tarasovn and Randolph 2011, Fig. 2, p. 889)

Publisher's Note Springer Nature remains neutral with regard to jurisdictional claims in published maps and institutional affiliations. 